\title{
THE HAUSDORFF DIMENSION OF SELF-SIMILAR SETS UNDER A PINCHING CONDITION
}

\author{
XIAOPING GU
}

(Communicated by Charles Pugh)

\begin{abstract}
We study self-similar sets in the case where the construction diffeomorphisms are not necessarily conformal. Using topological pressure we give an upper estimate of the Hausdorff dimension, when the construction diffeomorphisms are $C^{1+\kappa}$ and satisfy a $\kappa$-pinching condition for some $\kappa \leq 1$. Moreover, if the construction diffeomorphisms also satisfy the disjoint open set condition we then give a lower bound for the Hausdorff dimension.
\end{abstract}

\section{INTRODUCTION}

The construction of a self-similar set starts with a $k \times k$ matrix $A=\left(a_{i j}\right)$ which has entries zeros and ones, with all entries of $A^{N}$ positive for some $N>0$; see [H]. For each nonzero $a_{i j}$ we give a contraction map $\varphi_{i j}: R^{l} \rightarrow R^{l}$ with $\left\|\varphi_{i j}(x)-\varphi_{i j}(y)\right\| \leq c\|x-y\|$, where $c<1$ is a constant and we are using the Euclidean norm on $R^{l}$. Define the Hausdorff metric by

$$
d(E, F)=\inf \{\delta \mid d(x, F) \leq \delta \text { for all } x \in E \text {, and } d(y, E) \leq \delta \text { for all } y \in F\}
$$

in the space $\mathscr{C}$ of all nonempty compact subsets of $R^{l}$. See, for example, [H] or [F]. The map $\Phi$ on the $k$-fold product space $\mathscr{C}^{k}$ given by

$$
\Phi\left(F_{1}, \ldots, F_{l}\right)=\left(\bigcup_{j=1}^{k} \varphi_{1 j}\left(F_{j}\right), \ldots, \bigcup_{j=1}^{k} \varphi_{k j}\left(F_{j}\right)\right)
$$

is a contraction map. By the Banach Fixed Point Theorem the contraction map $\Phi$ has a unique fixed point in $\mathscr{C}^{k}$, i.e., a vector of compact nonempty subsets of $R^{l},\left(E_{1}, \ldots, E_{k}\right) \in \mathscr{C}^{k}$, with $\bigcup_{a_{i j}=1} \varphi_{i j}\left(E_{j}\right)=E_{i}$. The union $E=\bigcup_{i=1}^{k} E_{i}$ is called a self-similar set.

Let $\Sigma=\Sigma_{A}^{+}=\left\{\left(x_{0}, x_{1}, \ldots, x_{n}, \ldots\right) \mid 1 \leq x_{i} \leq k\right.$ and $a_{x_{i} x_{i+1}}=1$ for all $i \geq 0\}$ be the shift space with the following metric: for $\mathbf{x}=\left(x_{0}, x_{1}, \ldots\right)$, $\mathbf{y}=\left(y_{0}, y_{1}, \ldots\right)$ in $\Sigma, d(\mathbf{x}, \mathbf{y})=2^{-n}$ if and only if $n=\min \left\{m \mid x_{m} \neq y_{m}\right\}$. Let $\sigma$ be the shift map of $\Sigma$, and let $\pi: \Sigma \rightarrow E$ be given by

$$
\pi\left(x_{0}, x_{1}, \ldots, x_{n}, \ldots\right)=\text { the only point in } \bigcap_{n \geq 1} \varphi_{x_{0} x_{1}} \varphi_{x_{1} x_{2}} \cdots \varphi_{x_{n} x_{n+1}}\left(E_{x_{n+1}}\right) \text {. }
$$

Received by the editors May 27, 1991 and, in revised form, September 22, 1991.

1991 Mathematics Subject Classification. Primary 58F11, 28C10.

Partially supported by NSF Grant \#DMS 9101911. 
It is clear that $\pi$ is a Hölder continuous surjective map. We will denote the composition $\varphi_{x_{0} x_{1}} \cdots \varphi_{x_{n-1} x_{n}}$ by $\varphi_{x_{0} \cdots x_{n}}$. Also, we assume all $\varphi_{i j}$ to be $C^{1}$ diffeomorphisms and denote the derivative of $\varphi_{i j}$ at a point $x$ by $T_{x} \varphi_{i j}$ or $T \varphi_{i j}(x)$.

Definition 1.1. The $j$ th Lyapunov number of a linear map $L$, denoted by $\alpha_{j}(L)$, is the square root of the $j$ th largest eigenvalue of $L L^{*}$, where $L^{*}$ is the conjugate of $L$. Write $\omega_{t}(L)=\alpha_{1}(L) \cdots \alpha_{[t]}(L)_{\alpha_{[l]+1}}(L)^{t-[t]}$. For a set of construction diffeomorphisms $\left\{\varphi_{i j}\right\}$, define $\lambda_{t}: \Sigma \rightarrow R$ for each $t \in[0, l]$ and $\mathbf{x}=\left(x_{0} x_{1} \cdots\right) \in \Sigma$ by

$$
\begin{aligned}
\lambda_{t}(\mathbf{x})= & \log \alpha_{1}\left(T \varphi_{x_{0} x_{1}}(\pi \sigma \mathbf{x})\right)+\cdots+\log \alpha_{[t]}\left(T \varphi_{x_{0} x_{1}}(\pi \sigma \mathbf{x})\right) \\
& +(t-[t]) \log \alpha_{[t]+1}\left(T \varphi_{x_{0} x_{1}}(\pi \sigma \mathbf{x})\right) \\
= & \log \omega_{t}\left(T \varphi_{x_{0} x_{1}}(\pi \sigma \mathbf{x})\right) .
\end{aligned}
$$

Here "log" is the natural logarithm.

The constructions and dimensions of self-similar sets have been studied by several authors under various restrictions. In this paper we relax the restrictions on construction diffeomorphisms to a $\kappa$-pinching condition, which is defined as follows.

Definition 1.2. We say that a $C^{1}$ homeomorphism $\varphi_{i j}$ satisfies the $\kappa$-pinching condition if for all $x \in E$ the derivatives satisfy $\left\|T_{x} \varphi_{i j}\right\|^{1+\kappa} \cdot\left\|T_{\varphi_{i j}(x)} \varphi_{i j}^{-1}\right\|<1$.

Remark. If $T_{x} \varphi_{i j} T_{x} \varphi_{i j}^{*}$ has eigenvalues $\alpha_{1, i j}(x)^{2} \geq \cdots \geq \alpha_{l, i j}(x)^{2}$ where $T_{x} \varphi_{i j}^{*}$ denotes the conjugate of $T_{x} \varphi_{i j}$, the numbers $\alpha_{1, i j}(x), \ldots, \alpha_{l, i j}(x)$ are Lyapunov numbers with $1>\alpha_{1, i j}(x) \geq \cdots \geq \alpha_{l, i j}(x)>0$. The pinching condition is equivalent to $\alpha_{1, i j}(x)^{1+\kappa}<\alpha_{l, i j}(x)$.

For the definition and properties of Hausdorff dimension, refer to $[\mathrm{K}]$. Also, we use the definitions and notions of $[\mathrm{W}]$ in the discussion concerning topological pressure.

Theorem 1. Let $\left\{\varphi_{i j}\right\}$ be the $C^{1}$ construction diffeomorphisms for the selfsimilar set $E$, satisfying the $\kappa$-pinching condition for some positive number $\kappa \leq 1$. Suppose the derivatives of all $\left\{\varphi_{i j}\right\}$ are Hölder continuous of order $\kappa$. If $t$ is the unique positive number such that the topological pressure $P\left(\sigma, \lambda_{t}\right)=0$, then the Hausdorff dimension $\operatorname{HD}(E) \leq t$.

Let us recall the disjoint open set condition on the construction of self-similar sets; see $[\mathrm{H}]$. It states that for each integer $i$ from 1 to $k$ there is a nonempty open set $U_{i}$ such that

$$
\bigcup_{a_{i j}=1} \varphi_{i j}\left(U_{j}\right) \subset U_{i} \quad \text { and } \quad \varphi_{i j}\left(U_{j}\right) \cap \varphi_{i k}\left(U_{k}\right)=\varnothing \quad \text { if } j \neq k .
$$

For $n \geq 0$, denote $U_{n}(\mathbf{x})=\varphi_{x_{0} x_{1}} \varphi_{x_{1} x_{2}} \cdots \varphi_{x_{n-1} x_{n}}\left(U_{x_{n}}\right)$. It follows that $E_{i} \subset \bar{U}_{i}$ and that the collection $\left\{U_{n}(\mathbf{x}): \mathbf{x} \in \Sigma\right\}$ is pairwisely disjoint for each fixed $n$.

Theorem 2. Let $\left\{\varphi_{i j}\right\}$ be the $C^{1}$ construction diffeomorphisms for the selfsimilar set $E$, satisfying both the $\kappa$-pinching condition for some positive number $\kappa \leq 1$, and the disjoint open set condition. Suppose the derivatives of all $\left\{\varphi_{i j}\right\}$ are Hölder continuous of order $\kappa$. If $t$ is the unique positive number 
such that the topological pressure $P\left(\sigma, \lambda_{t}\right)=0$, then the Hausdorff dimension $\mathrm{HD}(E) \geq t /(1+\kappa)-l \kappa$.

Remark. We call a $C^{1}$ diffeomorphism $C^{1+\kappa}$ if its derivative is Hölder continuous of order $\kappa$. If we fix the construction to be $C^{1+\beta}$ for some $\beta>0$ but let $\kappa \rightarrow 0$ for the $\kappa$-pinching condition, then our upper and lower bounds will coincide with the estimate for conformal cases in [B1].

Theorem 1 is proved in $\S 2$, and Theorem 2 is proved in $\S 3$. As a corollary of Theorems 1 and 2 , in $\S 4$ we will also discuss some continuity in the $C^{1}$ topology of the Hausdorff dimension at conformal $C^{1+\kappa}$ constructions under disjoint open set condition. For discussions of the constructions of self-similar sets using similitudes and their dimensions, see $[\mathrm{H}]$ and $[\mathrm{MW}]$. For the constructions using "conformal" contraction maps, see [B1]. Other related works can be found in [D, B2, F]. A similar result for basic sets in two dimensions can be found in [MM].

\section{THE UPPER BOUND}

Lemma 2.1. If all construction diffeomorphisms $\varphi_{i j}$ are $C^{1+\kappa}$ and satisfy the $\kappa$-pinching condition, then for any $\varepsilon>0$, there exists $\delta>0$ depending only on $\varepsilon$, such that for all $x \in E$, all $a$ with $0<a<\delta$, and all $\mathbf{x}=\left(x_{0}, x_{1}, \ldots\right)$ in $\Sigma$, all integers $n>0$, we have

$$
\varphi_{x_{0} \cdots x_{n}} B(x, a) \subset \varphi_{x_{0} \cdots x_{n}}(x)+(1+\varepsilon)^{n} T_{x} \varphi_{x_{0} \cdots x_{n}} B(0, a) .
$$

Here $B(x, a)$ denotes a ball of radius a centering at $x$ in $R^{l}$.

Proof. Using Taylor's formula, for any $y, w \in R^{l}$,

$$
\varphi_{x_{0} x_{1}}(y+w)=\varphi_{x_{0} x_{1}}(y)+T_{y} \varphi_{x_{0} x_{1}}(w)+r_{x_{0} x_{1}}(w, y) .
$$

Since $E$ compact, we can find some constant $C>0$ and $c>0$, such that for all $y \in E$ and $w \in R^{l}$ with $\|w\| \leq c$, we have $\left\|r_{x_{0} x_{1}}(w, y)\right\|<C\|w\|^{1+\kappa}$. We will set also $b=\min _{x \in E, i, j}\left\{\alpha_{l, i j}(x)\right\}$, where $\alpha_{l, i j}$ is the square root of the least eigenvalue of $T_{x} \varphi_{i j} T_{x} \varphi_{i j}^{*}$.

Fix any small $\varepsilon>0$. Since $E$ is compact and all construction diffeomorphisms satisfy the $\kappa$-pinching condition, without loss of generality we can assume $\varepsilon$ to be so small that for all pairs $(i, j)$,

$$
\left\|(1+\varepsilon) T_{x} \varphi_{i j}\right\|<1 \text { for all } x \in E,
$$

$$
(1+\varepsilon)^{\kappa} \alpha_{1, i j}(x)^{1+\kappa}<\alpha_{l, i j}(x) \text { for all } x \in E .
$$

Pick $\delta>0$, with $\delta<\min \left\{c,(b \varepsilon / C)^{1 / \kappa}\right\}$. Thus $\delta^{\kappa}<\varepsilon \alpha_{l, i j}(x) / C$ for all $x \in E$ and all pairs of $(i, j)$. Let $a \leq \delta$, and pick any $w \in R^{l}$ with $\|w\|<a$. For any $x$ in $E,\left\|r_{x_{0} x_{1}}(x, w)\right\|<C\|w\|^{1+\kappa}<C a^{1+\kappa} \leq a C \delta^{\kappa} \leq a \varepsilon \alpha_{l, x_{0} x_{1}}(x)$ and thus $r_{x_{0} x_{1}}(w, x) \in \varepsilon \alpha_{l, x_{0} x_{1}}(x) B(0, a)$. Since

$$
\varepsilon \alpha_{l, x_{0} x_{1}}(x) B(0, a) \subset \varepsilon T_{x} \varphi_{x_{0} x_{1}} B(0, a),
$$

it follows from (2.2) that

$$
\begin{aligned}
\varphi_{x_{0} x_{1}}(x+w) & =\varphi_{x_{0} x_{1}}(x)+T_{x} \varphi_{x_{0} x_{1}}(w)+r_{x_{0} x_{1}}(w, x) \\
& \in \varphi_{x_{0} x_{1}}(x)+T_{x} \varphi_{x_{0} x_{1}} B(0, a)+\varepsilon T_{x} \varphi_{x_{0} x_{1}} B(0, a) \\
& =\varphi_{x_{0} x_{1}}(x)+(1+\varepsilon) T_{x} \varphi_{x_{0} x_{1}} B(0, a) .
\end{aligned}
$$


This gives (2.1) for $n=1$. Now the induction hypothesis gives

$$
\begin{aligned}
\varphi_{x_{0} x_{1} \cdots x_{n}} B(x, a) & =\varphi_{x_{0} x_{1}} \varphi_{x_{1} \cdots x_{n}} B(x, a) \\
& \subset \varphi_{x_{0} x_{1}}\left[\varphi_{x_{1} \cdots x_{n}}(x)+(1+\varepsilon)^{n-1} T_{x} \varphi_{x_{1} \cdots x_{n}} B(0, a)\right] .
\end{aligned}
$$

Using (2.2),

$$
\begin{aligned}
\varphi_{x_{0} x_{1}}[ & \left.\varphi_{x_{1} \cdots x_{n}}(x)+(1+\varepsilon)^{n-1} T \varphi_{x_{1} \cdots x_{n}}(w)\right] \\
= & \varphi_{x_{0} \cdots x_{n}}(x)+(1+\varepsilon)^{n-1} T \varphi_{x_{0} \cdots x_{n}}(w) \\
& +r_{x_{0} x_{1}}\left((1+\varepsilon)^{n-1} T \varphi_{x_{1} \cdots x_{n}}(w), \varphi_{x_{1} \cdots x_{n}}(x)\right) .
\end{aligned}
$$

Because of (2.3), $\left\|(1+\varepsilon)^{n-1} T \varphi_{x_{1} \cdots x_{n}}(w)\right\|<\|w\|<a$, where $w \in B(0, a)$. Using (2.4), we have

$$
\begin{aligned}
\| r_{x_{0} x_{1}} & \left((1+\varepsilon)^{n-1} T \varphi_{x_{1} \cdots x_{n}}(w), \varphi_{x_{1} \cdots x_{n}}(x)\right) \| \\
& <C\left\|(1+\varepsilon)^{n-1} T \varphi_{x_{1} \cdots x_{n}}(w)\right\|^{1+\kappa} \\
& \leq C(1+\varepsilon)^{(n-1)(1+\kappa)} \cdot\left[\alpha_{1, x_{1} x_{2}}\left(\varphi_{x_{2} \cdots x_{n}}(x)\right) \cdots \alpha_{1, x_{n-1} x_{n}}(x)\|w\|\right]^{1+\kappa} \\
& <C(1+\varepsilon)^{n-1} \alpha_{l, x_{1} x_{2}}\left(\varphi_{x_{2} \cdots x_{n}}(x)\right) \cdots \alpha_{l, x_{n-1} x_{n}}(x)\|w\|^{1+\kappa} \\
& <(1+\varepsilon)^{n-1} a^{\kappa} C \alpha_{l, x_{1} x_{2}}\left(\varphi_{x_{2} \cdots x_{n}}(x)\right) \cdots \alpha_{l, x_{n-1} x_{n}}(x)\|w\| \\
& <\varepsilon(1+\varepsilon)^{n-1} \alpha_{l, x_{0} x_{1}}\left(\varphi_{x_{1} \cdots x_{n}}(x)\right) \cdot \alpha_{l, x_{1} x_{2}}\left(\varphi_{x_{2} \cdots x_{n}}(x)\right) \cdots \alpha_{l, x_{n-1} x_{n}}(x) a .
\end{aligned}
$$

On the other hand $T_{x} \varphi_{i j} B(0, a) \supset \alpha_{l, i j}(x) B(0, a)$, and it follows that

$$
\operatorname{Tx} \varphi_{x_{0} \cdots x_{n}} B(0, a) \supset \alpha_{l, x_{0} x_{1}}\left(\varphi_{x_{1} \cdots x_{n}}(x)\right) \cdots \alpha_{l, x_{n-1} x_{n}}(x) B(0, a) .
$$

Hence $r_{x_{0} x_{1}}\left((1+\varepsilon)^{n-1} T \varphi_{x_{1} \cdots x_{n}}(w), \varphi_{x_{1} \cdots x_{n}}(x)\right) \in \varepsilon(1+\varepsilon)^{n-1} T_{x} \varphi_{x_{0} \cdots x_{n}} B(0, a)$. Therefore,

$$
\begin{aligned}
\varphi_{x_{0} x_{1}} & {\left[\varphi_{x_{1} \cdots x_{n}}(x)+(1+\varepsilon)^{n-1} T \varphi_{x_{1} \cdots x_{n}}(w)\right] } \\
& \in \varphi_{x_{0} \cdots x_{n}}(x)+(1+\varepsilon)^{n-1} T_{x} \varphi_{x_{0} \cdots x_{n}} B(0, a)+\varepsilon(1+\varepsilon)^{n-1} T_{x} \varphi_{x_{0} \cdots x_{n}} B(0, a) \\
& \subset \varphi_{x_{0} \cdots x_{n}}(x)+(1+\varepsilon)^{n} T_{x} \varphi_{x_{0} \cdots x_{n}} B(0, a) .
\end{aligned}
$$

Thus (2.1) is true for $n$. This completes the induction process.

I have learned that Jiang $[\mathrm{J}]$ has a distorsion lemma for a regular nonconformal semigroup, which is a semigroup of pinched contracting diffeomorphisms. His version is stronger than our version here. However, for our purpose of estimating Hausdorff dimensions, our version is strong enough.

Proposition 2.2. If all construction diffeomorphisms $\varphi_{i j}$ are $C^{1+\kappa}$ and satisfy the $\kappa$-pinching condition where $0<\kappa \leq 1$, and if the topological pressure $P\left(\sigma, \lambda_{t}\right)<0$ where $\sigma$ is the shift map in $\Sigma$, then the Hausdorff dimension $\mathrm{HD}(E) \leq t$.

Proof. Choose small $\varepsilon>0$ with $P\left(\sigma, \lambda_{t}\right)<-2 t \varepsilon$, satisfying both $(2.3)$ and (2.4). By Lemma 2.1, there exists $\delta>0$ such that $(2.1)$ holds for all integer $n>0$ and each $x \in E$, when $0<a<\delta$.

We fix $a<\delta$ small enough, and a positive integer $n$ big enough, such that (see [W] for notation) $\log P_{n}\left(\sigma, \lambda_{t}, a\right)<-2 n t \varepsilon$. Recall that $\pi$ is Hölder continuous. Suppose that $\gamma$ is the exponent such that there exists a constant $D$ 
with $|\pi(\mathbf{x})-\pi(\mathbf{y})|<D \cdot d(\mathbf{x}, \mathbf{y})^{\gamma}$ for all $\mathbf{x}, \mathbf{y}$ in $\Sigma$. Fix $a^{\prime}<\min \left\{D^{-1 / \gamma} a^{1 / \gamma}, a\right\}$. Pick $m$ with $2^{-m-1}<a^{\prime} \leq 2^{-m}$. Let

$$
K^{\prime}=\left\{\left(x_{0}, \ldots, x_{m+n}\right) \mid \text { there exists } \mathbf{x} \in \Sigma \text { with } \mathbf{x}=\left(x_{0}, \ldots, x_{m+n}, \ldots\right)\right\} .
$$

Choose for each word $\left(x_{0}, \ldots, x_{m+n}\right)$ in $K^{\prime}$ a point $\mathbf{x}$ in $\Sigma$ with the initial of $x_{0}, \ldots, x_{m+n}$ to form a subset $K$ of $\Sigma$. The subset $K$ is $\left(n, a^{\prime}\right)$ separated and is maximal in the sense that one cannot add another point to $K$ such that it is still $\left(n, a^{\prime}\right)$ separated. Thus, the collection $\left\{\sigma^{-n} B\left(\sigma^{n} \mathbf{x}, a^{\prime}\right) \mid \mathbf{x} \in K\right\}$ is an open cover for $\Sigma$. Notice that $\pi \mathbf{x}=\varphi_{x_{0} x_{1}} \pi \sigma \mathbf{x}$. Since $\pi B\left(\mathbf{x}, a^{\prime}\right) \subset B(\pi(\mathbf{x}), a)$ and $\pi\left\{\sigma^{-n} B\left(\sigma^{n} \mathbf{x}, a^{\prime}\right) \mid \mathbf{x} \in K\right\} \subset\left\{\varphi_{x_{0} x_{1} \cdots x_{n}} B\left(\pi \sigma^{n} \mathbf{x}, a\right) \mid \mathbf{x}=\left(x_{0}, x_{1}, \ldots, x_{n} \ldots\right) \in\right.$ $K\}$ follows, $\left\{\varphi_{x_{0} x_{1} \cdots x_{n}} B\left(\pi \sigma^{n} \mathbf{x}, a\right) \mid \mathbf{x}=\left(x_{0}, x_{1}, \ldots, x_{n} \ldots\right) \in K\right\}$ is an open cover for $E=\bigcup_{i=1}^{l} E_{i}$.

Using (2.1) of Lemma 2.1,

$$
\varphi_{x_{0} \cdots x_{n}} B\left(\pi \sigma^{n} \mathbf{x}, a\right) \subset \varphi_{x_{0} \cdots x_{n}}\left(\pi \sigma^{n} \mathbf{x}\right)+(1+\varepsilon)^{n} T_{\pi \sigma^{n}} \mathbf{x} \varphi_{x_{0} \cdots x_{n}} B(0, a) .
$$

The right side of (2.5) is an ellipsoid with axes $\left\{a(1+\varepsilon)^{n} \alpha_{j}\left(T_{\pi \sigma^{n}} \mathbf{X} \varphi_{x_{0} \cdots x_{n}}\right) \mid 1 \leq\right.$ $j \leq l\}$. Pick $j$ with $j-1 \leq t<j$. Then that ellipsoid can be covered by

$$
\begin{gathered}
C \cdot \alpha_{1}\left(T_{\pi \sigma^{n} \mathbf{X}} \varphi_{x_{0} \cdots x_{n}}\right) \cdots \alpha_{j}\left(T_{\pi \sigma^{n}} \mathbf{X} \varphi_{x_{0} \cdots x_{n}}\right) / \alpha_{j}\left(T_{\pi \sigma^{n}} \mathbf{X} \varphi_{x_{0} \cdots x_{n}}\right)^{j} \\
\quad=C \cdot \omega_{j-1}\left(T_{\pi \sigma^{n}} \mathbf{X} \varphi_{x_{0} \cdots x_{n}}\right) \alpha_{j}^{-j+1}\left(T_{\pi \sigma^{n}} \mathbf{X} \varphi_{x_{0} \cdots x_{n}}\right)
\end{gathered}
$$

balls of radius $a(1+\varepsilon)^{n} \alpha_{j}\left(T_{\pi \sigma^{n}} \mathbf{x} \varphi_{x_{0} \cdots x_{n}}\right)$, where the constant $C>0$ depends only on the dimension of $R^{l}$. Now we calculate the Hausdorff $t$-measure of $E$, using the smaller balls of radius $a(1+\varepsilon)^{n} \cdot \alpha_{j}\left(T_{\pi \sigma^{n}} \mathbf{X} \varphi_{x_{0} \cdots x_{n}}\right)<a$ to cover the open set $\varphi_{x_{0} \cdots x_{n}} B\left(\pi \sigma^{n} \mathbf{x}, a\right)$. If $\left\{P_{i}: i \in I\right\}$ is an open cover for $E$ where $P_{i}$ is a ball of radius $r_{i}$, then we define $|I|=\max _{i \in I} r_{i}$ and $\mu(a, t)=\inf _{|I|<a} \sum_{i \in I} r_{i}^{t}$. We have

$$
\begin{aligned}
& \mu(a, t) \leq \sum_{\mathbf{x} \in K} C \omega_{j-1}\left(T_{\pi \sigma^{n}} \mathbf{X} \varphi_{x_{0} \cdots x_{n}}\right) \alpha_{j}^{-j+1} \\
& \times\left(T_{\pi \sigma^{n}} \mathbf{X} \varphi_{x_{0} \cdots x_{n}}\right)\left[a(1+\varepsilon)^{n} \alpha_{j}\left(T_{\pi \sigma^{n}} \mathbf{X} \varphi_{x_{0} \cdots x_{n}}\right)\right]^{t} \\
& =(1+\varepsilon)^{n t} a^{t} C \sum_{\mathbf{x} \in K} \omega_{t}\left(T_{\pi \sigma^{n}} \mathbf{x} \varphi_{x_{0} \cdots x_{n}}\right) \\
& \leq(1+\varepsilon)^{n t} C \sum_{\mathbf{x} \in K} \omega_{t}\left(T_{\pi \sigma \mathbf{x}} \varphi_{x_{0} x_{1}}\right) \omega_{t}\left(T_{\pi \sigma^{2}} \mathbf{x} \varphi_{x_{1} x_{2}}\right) \cdots \omega_{t}\left(T_{\pi \sigma^{n} \mathbf{x}} \varphi_{x_{n-1} x_{n}}\right) \\
& =(1+\varepsilon)^{n t} C \sum_{\mathbf{x} \in K} \exp \left[\lambda_{t}(\mathbf{x})+\lambda_{t}(\sigma \mathbf{x})+\cdots+\lambda_{t}\left(\sigma^{n-1} \mathbf{x}\right)\right] \\
& \leq(1+\varepsilon)^{n t} C P_{n}\left(\sigma, \lambda_{t}, a^{\prime}\right) \leq(1+\varepsilon)^{n t} C P_{n}\left(\sigma, \lambda_{t}, a\right) \\
& \leq C \exp (n t \varepsilon) \exp (-2 n t \varepsilon) \rightarrow 0 \text {, }
\end{aligned}
$$

as $n \rightarrow \infty$. Thus $\mu(a, t)=0$. Since $a$ can be arbitrarily small, $\mu(t)=0$. It follows that $\operatorname{HD}(E) \leq t$.

Proof of Theorem 1. $P\left(\sigma, \lambda_{t}\right)$ is a decreasing function of $t$ 's since $E$ is compact and $\lambda_{t}$ is strictly decreasing with respect to $t$. So there is only one real number $t$ such that $P\left(\sigma, \lambda_{t}\right)=0$. Also, the unique $t$ with $P\left(\sigma, \lambda_{t}\right)=0$ is equal to $\inf \left\{t: P\left(\sigma, \lambda_{t}\right)<0\right\}$. Consequently, we have $\operatorname{HD}(E) \leq t$ where $P\left(\sigma, \lambda_{t}\right)=0$. 


\section{THE LOWER BOUND}

Proof of Theorem 2. Notice that for each $t$, the map $\lambda_{t}$ is Hölder continuous on $\Sigma$. So there exists an equilibrium state $\mu$ for $\lambda_{t}$, in the sense that

$$
P\left(\sigma, \lambda_{t}\right)=h_{\mu}(\sigma)+\int \lambda_{t} d \mu .
$$

Fix any $\rho>0$, let us estimate the $\mu$-measure of a ball $B(z, \rho)$ centered at $z$ with radius $\rho$. For each $\mathbf{x} \in \Sigma$ choose the unique $n=n(\mathbf{x}) \geq 0$ such that the diameters satisfy

$$
\operatorname{diam}\left(U_{n}(\mathbf{x})\right) \leq \rho<\operatorname{diam}\left(U_{n-1}(\mathbf{x})\right) .
$$

Lemma 3.1. There exists a constant $c>0$ such that for all $\mathbf{x} \in \Sigma$, the open set $U_{n(\mathbf{x})}(\mathbf{x})$ is contained in a ball of radius $\rho$ and contains a ball of radius $c \rho^{1+\kappa}$.

Proof. It is clear that $U_{n}(\mathbf{x})$ is contained in a ball of radius $\rho$. Since the radius of $U_{n}(\mathbf{x})$ decreases to 0 as $n$ grows to infinity, without loss of generality we can assume the maximum diameter $R$ of all $U_{i}$ is less than the number $\delta$ given in Lemma 2.1. Also pick $r$ small enough that each $U_{i}$ contains a ball of radius $r$. Then $U_{n}(\mathbf{x})$ contains a ball of radius

$$
r \cdot \alpha_{l, x_{0} x_{1}}(\pi \sigma \mathbf{x}) \cdots \alpha_{l, x_{n-1} x_{n}}\left(\pi \sigma^{n} \mathbf{x}\right)>r \cdot \alpha_{1, x_{0} x_{1}}^{1+\kappa}(\pi \sigma \mathbf{x}) \cdots \alpha_{1, x_{n-1} x_{n}}^{1+\kappa}\left(\pi \sigma^{n} \mathbf{x}\right) .
$$

But on the other hand

$$
\rho \leq \operatorname{diam}\left(U_{n-1}(\mathbf{x})\right) \leq \alpha_{1, x_{0} x_{1}}(\pi \sigma \mathbf{x}) \cdots \alpha_{1, x_{n-2} x_{n-1}}\left(\pi \sigma^{n-1} \mathbf{x}\right) R,
$$

which implies that $\alpha_{1, x_{0} x_{1}}(\pi \sigma \mathbf{x}) \cdots \alpha_{1, x_{n-1} x_{n}}\left(\pi \sigma^{n} \mathbf{x}\right) \geq \alpha_{1} \rho / R$ where the constant $\alpha_{1}=\min _{y \in E, i, j}\left\{\alpha_{1, i j}(y)\right\}>0$ does not depend on either $n$ or $\mathbf{x}$. Therefore $U_{n}(\mathbf{x})$ contains a ball of radius $>r \rho^{1+\kappa} \alpha_{1}^{1+\kappa} / R^{1+\kappa}$. Writing $c=$ $\alpha_{1}^{1+\kappa} r / R^{1+\kappa}$ a constant, $U_{n}(\mathbf{x})$ contains a ball of radius $c \rho^{1+\kappa}$ as desired.

For two points $\mathbf{x}, \mathbf{y} \in \Sigma$, since the construction maps satisfy the open set condition, $U_{n(\mathbf{x})}(\mathbf{x})$ and $U_{n(\mathbf{y})}(\mathbf{y})$ are either equal or disjoint. Let $\Gamma \subset \Sigma$ be a subset such that $\left\{U_{n(\mathbf{x})}(\mathbf{x}) \mid \mathbf{x} \in \Gamma\right\}$ is a disjoint collection which contains all $U_{n(\mathbf{x})}(\mathbf{x})$ for $\mathbf{x} \in \Sigma$. Notice that $\left\{\bar{U}_{n(\mathbf{x})}(\mathbf{x}) \mid \mathbf{x} \in \Gamma\right\}$ covers $E$.

Lemma 3.2 (similar to $[\mathrm{H}, 5.3(\mathrm{a})])$. At most $3^{l} c^{-l} \rho^{-\kappa l}$ of $\left\{\bar{U}_{n(\mathbf{x})}(\mathbf{x}) \mid \mathbf{x} \in \Gamma\right\}$ can meet $B(z, \rho)$.

Proof. Suppose that $\bar{V}_{1}, \ldots, \bar{V}_{m}$ in $\left\{\bar{U}_{n(\mathbf{x})}(\mathbf{x}) \mid \mathbf{x} \in \Gamma\right\}$ meet $B(z, \rho)$. Then each of them is a subset of $B(z, 3 \rho)$. By the definition of $\Gamma$ the sets in the collection $\left\{\bar{U}_{n(\mathbf{x})}(\mathbf{x}) \mid \mathbf{x} \in \Gamma\right\}$ are disjoint. Comparing the volumes we have $m J c^{l} \rho^{l(1+\kappa)} \leq J 3^{l} \rho^{l}$ where $J$ is the volume of a unit ball in $R^{l}$. Hence $m \leq 3^{l} c^{-l} \rho^{-\kappa l}$.

Let $C_{n}(\mathbf{x})=\left\{\mathbf{y}=\left(y_{0}, y_{1}, \ldots\right) \in \Sigma \mid y_{0}=x_{0}, \ldots, y_{n}=x_{n}\right\}$ be the $n$ cylinder. Recall that $\mu$ is a Gibbs measure (see [Bo] for a discussion or [B2] for a summary). There exists a constant $d>0$, with

$\mu\left(C_{n}(\mathbf{x})\right) \in\left[d^{-1}, d\right] \cdot \exp \left(-P\left(\sigma, \lambda_{t}\right) n+S_{n} \lambda_{t}(\mathbf{x})\right), \quad$ for each cylinder $C_{n}(\mathbf{x})$ in $\Sigma$. 
Thus $\mu\left(C_{n}(\mathbf{x})\right) \in\left[d^{-1}, d\right] \cdot \exp \left(S_{n} \lambda_{t}(\mathbf{x})\right)$, since $P\left(\sigma, \lambda_{t}\right)=0$. So,

$$
\begin{aligned}
\mu\left(C_{n}(\mathbf{x})\right) & \leq d \exp S_{n} \lambda_{t}(\mathbf{x}) \\
& \leq d\left[\alpha_{1, x_{0} x_{1}}(\pi \sigma \mathbf{x}) \cdots \alpha_{1, x_{n-1} x_{n}}\left(\pi \sigma^{n} \mathbf{x}\right)\right]^{t} \\
& \leq d\left[\alpha_{l, x_{0} x_{1}}(\pi \sigma \mathbf{x}) \cdots \alpha_{l, x_{n-1} x_{n}}\left(\pi \sigma^{n} \mathbf{x}\right)\right]^{t /(1+\kappa)} \\
& \leq d \cdot\left[\operatorname{diam}\left(U_{n(\mathbf{x})} / r\right]^{t /(1+\kappa)} .\right.
\end{aligned}
$$

Hence if $n=n(\mathbf{x})$ we obtain

$$
\mu\left(C_{n(\mathbf{x})}(\mathbf{x})\right) \leq \frac{d \rho^{t /(1+\kappa)}}{r^{t /(1+\kappa)}} .
$$

Noticing $\pi C_{n}(\mathbf{x}) \supset \bar{U}_{n}(\mathbf{x}) \cap E$, by Lemma 3.2,

$$
\pi_{*} \mu(B(z, \rho)) \leq\left[3^{l} c^{-l} d r^{-t /(1+\kappa)}\right] \rho^{t /(1+\kappa)-l \kappa}
$$

By the Frostman lemma (see $[\mathrm{K}]$ for a proof), $\mathrm{HD}(E) \geq t /(1+\kappa)-l \kappa$.

\section{Some CONTINUity OF THE HAUSDORFF Dimension in $C^{1}$ TOPOLOGY}

The construction of the self-similar set $E_{\varphi}$ depends on the contracting diffeomorphisms $\left\{\varphi_{i j}\right\}$. Now let us fix $0<\beta \leq 1$, and consider a $C^{1}$ perturbation to a $C^{1+\beta}$ conformal construction with diffeomorphisms $\left\{\varphi_{i j}\right\}$, and obtain another matrix of contracting diffeomorphisms $\left\{\psi_{i j}\right\}$, which is not necessarily conformal. Denote the new self-similar set for $\psi$ by $E_{\psi}$. Define $d_{C^{1}}(\varphi, \psi)=\max _{i, j} d_{C^{1}}\left\{\left(\varphi_{i j}, \psi_{i j}\right)\right\}$, where the latter $d_{C^{1}}$ is the $C^{1}$ metric. Note that for any $\kappa<\beta$, when $\psi$ is sufficiently $C^{1}$ close to $\varphi, \psi$ must be $C^{1+\kappa}$ and also $\kappa$-pinched. The following theorem is a corollary of Theorems 1 and 2 , which states that at a $C^{1+\beta}$ conformal construction satisfying the open set condition for self-similar sets, the Hausdorff dimension $\operatorname{HD}\left(E_{\psi}\right)$ depends continuously on $\left\{\psi_{i j}\right\}$ in $C^{1}$ topology.

Theorem 4.1. Let $\left\{\varphi_{i j}\right\}$ be a matrix of $C^{1+\beta}$ conformal construction diffeomorphisms for the self-similar set $E_{\varphi}$, satisfying the open set condition. For any $\varepsilon>0$, there exists $\delta>0$, such that for any $C^{1+\beta}$ construction $\psi$ satisfying the open set condition, with $d_{C^{1}}(\varphi, \psi)<\delta$, we have $\left|\operatorname{HD}\left(E_{\varphi}\right)-\operatorname{HD}\left(E_{\psi}\right)\right|<\varepsilon$.

Proof. Let $\lambda_{\varphi, s}(\mathbf{x})=\log \omega_{s}\left(T \varphi_{x_{0} x_{1}}(\pi \sigma \mathbf{x})\right)$ and $\lambda_{\psi, s}(\mathbf{x})=\log \omega_{s}\left(T \psi_{x_{0} x_{1}}(\pi \sigma \mathbf{x})\right)$ be two real functions on $\Sigma$ as defined in Definition 1.1. Let $t$ be such that $P\left(\sigma, \lambda_{\varphi, t}\right)=0$. Because $\varphi_{i j}$ 's are conformal, the Hausdorff dimension of $E_{\varphi}$ equals $t$. Also, remark that $P\left(\sigma, \lambda_{\varphi, t+\varepsilon}\right)<0$ for any $\varepsilon>0$.

Now fix any $\varepsilon>0$. Let $\kappa=\min \{\beta, \varepsilon / 4 l\}$ and let

$$
\varepsilon^{\prime}=\frac{1}{2} \min \left\{-P\left(\sigma, \lambda_{\varphi, t+\varepsilon}\right), P\left(\sigma, \lambda_{\varphi, t-\varepsilon / 4}\right)\right\}>0 .
$$

Since $\varphi$ is $C^{1+\beta}$ and conformal, there is $\delta>0$ such that a $C^{1+\beta}$ diffeomorphism $\psi$ is $C^{1+\kappa}$ and $\kappa$-pinched with $\left|\lambda_{\varphi, s}(\mathbf{x})-\lambda_{\psi, s}(\mathbf{x})\right|<\varepsilon^{\prime}$ for all $s \in[0, l]$, if $d_{C^{1}}(\varphi, \psi)<\delta$.

Then $P\left(\sigma, \lambda_{\psi, t+\varepsilon}\right)<P\left(\sigma, \lambda_{\varphi, t+\varepsilon}+\varepsilon^{\prime}\right) \leq P\left(\sigma, \lambda_{\varphi, t+\varepsilon}\right)+\varepsilon^{\prime}<0$. So $\operatorname{HD}\left(E_{\psi}\right) \leq$ $t+\varepsilon=\operatorname{HD}\left(E_{\varphi}\right)+\varepsilon$, by Proposition 2.2.

On the other hand, by $(4.1)$, when $d_{C^{1}}(\varphi, \psi)<\delta$, we have

$$
P\left(\sigma, \lambda_{\psi, t-\varepsilon / 4}\right)>P\left(\sigma, \lambda_{\varphi, t-\varepsilon / 4}-\varepsilon^{\prime}\right) \geq P\left(\sigma, \lambda_{\varphi, t-\varepsilon / 4}\right)-\varepsilon^{\prime}>0 .
$$


So we have some $s>t-\varepsilon / 4$ with $P\left(\sigma, \lambda_{\psi, s}\right)=0$ since $P\left(\sigma, \lambda_{\psi, s}\right)$ is strictly decreasing with respect to $s$. By Theorem $2, \operatorname{HD}\left(E_{\psi}\right) \geq s /(1+\kappa)-l \kappa \geq$ $s /(1+\varepsilon / 4 l)-l(\varepsilon / 4 l)>s(1-\varepsilon / 4 l)-\varepsilon / 4>s-\varepsilon / 2>t-\varepsilon$. It then follows that $\mathrm{HD}\left(E_{\psi}\right) \geq t-\varepsilon=\operatorname{HD}\left(E_{\varphi}\right)-\varepsilon$.

We say a construction $\varphi$ with diffeomorphisms $\left\{\varphi_{i j}\right\}$ satisfies the strong open set condition if there are open sets $U_{1}, \ldots, U_{l}$ in $R^{l}$ with $\varphi_{i j}\left(\bar{U}_{j}\right) \subset U_{i}$ for all $i, j$. If the construction $\varphi$ satisfies the strong open set condition, then $\psi$ must also satisfy the strong open set condition if it is $C^{1}$ close enough to $\varphi$. Thus we have obtained an immediate corollary of the above Theorem 4.1:

Corollary 4.2. Let $\left\{\varphi_{i j}\right\}$ be a matrix of $C^{1+\beta}$ conformal construction diffeomorphisms for the self-similar set $E_{\varphi}$, satisfying the strong open set condition. For any $\varepsilon>0$, there exists $\delta>0$, such that for any $C^{1+\beta}$ construction $\psi$ with $d_{C^{1}}(\varphi, \psi)<\delta$, we have $\left|\operatorname{HD}\left(E_{\varphi}\right)-\operatorname{HD}\left(E_{\psi}\right)\right|<\varepsilon$.

Finally we have a remark on the continuity of the Hausdorff dimension in $C^{1}$ topology at nonconformal constructions.

Remark 4.3. The following example shows that if the "conformal" condition for the construction diffeomorphisms $\left\{\varphi_{i j}\right\}$ fails, then the results in Theorem 4.1 and Corollary 4.2 can be false. The example is derived from Example 9.10 of Falconer [F, pp. 127-128].

Let $S, T_{\lambda}: R^{2} \rightarrow R^{2}$ be given by

$$
S(x, y)=(x / 2, y / 3+2 / 3), \quad T_{\lambda}(x, y)=(x / 2+\lambda, y / 3)
$$

where $\lambda \in[0,1 / 2)$ and $(x, y) \in R^{2}$. Let $\varphi_{11}=\varphi_{21}=S, \varphi_{12}=\varphi_{22}=T_{0}$. Take $\psi_{\lambda}=\left\{\psi_{i j, \lambda}\right\}$ where $\psi_{11, \lambda}=\psi_{21, \lambda}=S$ and $\psi_{12, \lambda}=\psi_{22, \lambda}=T_{\lambda}$. The strong open set condition is met for $\left\{\varphi_{i j}\right\}$. In fact, if we let $U_{1}=U_{2}=$ $(-1 / 8,9 / 8)^{2} \subset R^{2}$ then $\varphi_{i j}\left(\bar{U}_{j}\right) \subset U_{i}$.

Let $E_{\varphi}, E_{\psi_{\lambda}}$ be the self-similar sets for $\varphi$ and $\psi_{\lambda}$. Considering the projection of $E_{\psi_{\lambda}}$ to the $x$-axis, one knows that $\operatorname{HD}\left(E_{\psi_{\lambda}}\right) \geq 1$ for $\lambda>0$. But $E_{\varphi}$ is a Cantor set contained in the $y$-axis with the Hausdorff dimension $\operatorname{HD}\left(E_{\varphi}\right)=(\log 2) / \log 3<1$. Since $d_{C^{1}}\left(\psi_{\lambda}, \varphi\right)=\lambda$, letting $\lambda \rightarrow 0$ we know the Hausdorff dimension is not continuous at $\varphi$. We notice that $\left\{\varphi_{i j}\right\}$ are not conformal although $\left\{\varphi_{i j}\right\}$ and $\left\{\psi_{i j, \lambda}\right\}$ are all $3 / 4$ pinched.

It is a pleasure to thank Albert Fathi for his encouraging and helpful advice.

\section{REFERENCES}

[B1] T. Bedford, Hausdorff dimension and box dimension in self similar sets, Proc. Conf. Topology and Measure V, Greifswald, 1988, pp. 17-26.

[B2] , Dimension and dynamics for fractal recurrent sets, J. London Math. Soc. (2) 33 (1986), 89-100.

[Bo] R. Bowen, Equilibrium states and the ergodic theory of Anosov diffeomorphisms, Lecture Notes in Math., vol. 470, Springer, Berlin, 1975.

[D] F. M. Dekking, Recurrent sets, Adv. in Math. 44 (1982), 78-104.

[F] K. Falconer, Fractal geometry, mathematical foundations and applications, Wiley, England, 1990.

[H] J. E. Hutchinson, Fractals and self similarity, Indiana Univ. Math. J. 30 (1981), 713-747.

[J] Y. Jiang, On non-conformal semigroups, preprint, State University of New York at Stony Brook. 
[K] J. P. Kahane, Some random series of functions, second ed., Cambridge Univ. Press, Cambridge and New York, 1985.

[MM] H. McCluskey and A. Manning, Hausdorff dimension for horseshoes, Ergodic Theory Dynamical Systems 3 (1983), 251-260.

[MW] R. D. Mauldin and S. C. Williams, Hausdorff dimension in graph directed constructions, Trans. Amer. Math. Soc. 309 (1988), 811-829.

[W] P. Walters, An introduction to ergodic theory, Springer-Verlag, New York, Heidelberg, and Berlin, 1982.

Department of Mathematics, University of Florida, Gainesville, Florida 32611

E-mail address: xgu@math.ufl.edu 